\title{
Effects of the pairing energy on nuclear radii
}

\author{
C. Weber ${ }^{1,2}$, G. Audi ${ }^{3}$, D. Beck ${ }^{1}$, K. Blaum ${ }^{1,2}$, G. Bollen ${ }^{4}$, F. Herfurth ${ }^{1}$, H.-J. Kluge ${ }^{1}$, D. Lunney ${ }^{3}$, and S. Schwarz ${ }^{4}$ \\ 1 Gesellschaft für Schwerionenforschung m.b.H. - Darmstadt, Planckstr. 1, D-64291 Darmstadt, Germany \\ 2 Institute of Physics, Johannes Gutenberg-University, D-55099 Mainz, Germany \\ 3 CSNSM-IN2P3/CNRS, Université de Paris-Sud, F-91405 Orsay, France \\ 4 NSCL, Michigan State University, East Lansing MI 48824-1321, USA
}

Received: date / Revised version: date

\begin{abstract}
The mass region around the $Z=82$ shell closure is characterized by strong nuclear structure effects, like e.g. shape coexistence. In this contribution results from mass spectrometry and laser spectroscopy are examined for a possible correlation between mass values and radii.
\end{abstract}

PACS. 07.75.+h Mass spectrometers - 27.80.+w 190(less-than-or-equal-to)A(less-than-or-equal-to)219

This work was initiated at the ENAM 2001 conference based on the high-precision mass measurements of neutron-deficient $\mathrm{Hg}$ isotopes [1]. The appearance of shape coexistence as observed in the large odd-even staggering of $\mathrm{Hg}$ radii near the $N=104$ mid-shell region was explained by the size of the neutron pairing energy. As this quantity has an absolute value of only about $1 \mathrm{MeV}$, mass data available at that time were of insufficient precision for any analysis. The high relative mass uncertainty of $\delta m / m=10^{-8}$ and a resolving power of up to $10^{7}$ obtained with the ISOLTRAP Penning trap mass spectrometer is a necessary requirement to resolve isomeric states in the recent measurements on neutron-deficient $\mathrm{Tl}, \mathrm{Pb}$, and $\mathrm{Bi}$ isotopes [2]. New results of laser spectroscopy studies are available for neutron-deficient $\mathrm{Pb}$ isotopes [3] which will be compared with the $\mathrm{Pb}$ masses, in an upcoming work. The systematic comparison of the neutron pairing gap energies

$$
\begin{gathered}
\Delta^{3}(N)=\frac{(-1)^{N}}{2}[B(N-1)+B(N+1)-2 B(N)], \\
\Delta^{4}(N)=\frac{1}{2}\left[\Delta^{3}(N)+\Delta^{3}(N-1)\right]
\end{gathered}
$$

to the behaviour of the nuclear mean square charge radii $\left.\delta<r^{2}\right\rangle$ (data from $[4,5]$ ) is used to search for a possible correlation. Fig. 1 shows the two examples of mercury- and thallium-isotopes. In $\Delta^{3}(N)$ and $\Delta^{4}(N)$ the shell closure at $N=126$ is visible. In addition, distinct minima are observed around the mid-neutron shell. The systematic study of these fine correlations has become possible due to the high precision of the ISOLTRAP data.

\section{References}

1. S. Schwarz et al., Nucl. Phys. A 693, (2001) 533-545.
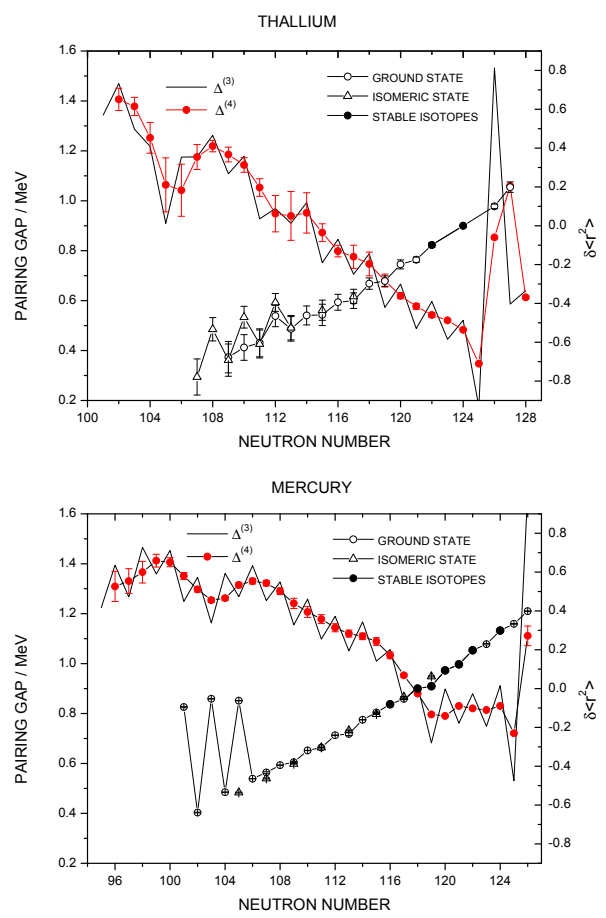

Fig. 1. Comparison of neutron pairing gap energies $\Delta^{(3)}, \Delta^{(4)}$ to nuclear mean square charge radii $\delta<r^{2}>$. Note that in thallium the radii of the isomer exhibit the staggering behaviour.

2. C. Weber, Ph.D. thesis, University of Heidelberg, 2003.

3. H. De Witte, Ph.D. thesis, University of Leuven, 2004 and H. De Witte, this issue.

4. G. Audi, A.H. Wapstra, C. Thibault, Nucl. Phys. A 729, (2003) 337-676.

5. J. Kluge, W. Nörtershäuser, Spectroc. Acta B 58, (2004) 1031-1045, and references therein. 